\title{
Simplification of State Transition Diagrams in Average Unavailability Analysis by Using Generalized Perturbation Theory
}

\author{
R. C. Vicente, F. C. Silva, P. F. Frutuoso e Melo, and A. C. M. Alvim \\ Graduate Program of Nuclear Engineering, Federal University of Rio de Janeiro, Avenida Horácio Macedo 2030, \\ Bloco G, Sala 206, 21941-914 Rio de Janeiro, RJ, Brazil
}

Correspondence should be addressed to A. C. M. Alvim; aalvim@gmail.com

Received 30 October 2013; Revised 21 February 2014; Accepted 21 February 2014; Published 17 April 2014

Academic Editor: Borut Mavko

Copyright (C) 2014 R. C. Vicente et al. This is an open access article distributed under the Creative Commons Attribution License, which permits unrestricted use, distribution, and reproduction in any medium, provided the original work is properly cited.

Safety analysis studies in nuclear engineering, more specifically system reliability, usually handle a great number of components, so that computational difficulties may arise. To face the problem of many component systems a method for simplifying the state transition diagram in Markovian reliability analyses has been proposed, using the edges which can be cut, since these latter have a smaller influence on system failure probability. In order to extend the application of GPT (Generalized Perturbation Theory), this work uses GPT formalism to reduce the number of states in a transition diagram, not considering the state probability as the integral quantity of interest, but the mean system unavailability instead. Therefore, after simplifying the original diagram, the mean unavailability for the new system was calculated and the results were very close to those of the original diagram integral quantity (giving a relative error of about $2 \%$ ), showing that the proposed simplification is quite reasonable and simple to apply.

\section{Introduction}

Computational resources available are an important element to be considered in system reliability analysis of large industrial facilities when working with systems with a large number of components. The computational capacity may be a factor of interest because of the need, in many cases, to consider dependencies between failure events, besides the large number of system states to be considered.

It is well known that the solution of a Markovian reliability model for obtaining time-dependent and steady-state system probabilities can be simplified if certain sets of states can be combined to form single states [1]. This lumpability or mergeability feature is further discussed in $[2,3]$.

References $[4,5]$ discuss this feature and present practical applications in which system symmetries are explored. These symmetries give rise to superstates which result from symmetrical states merging. One possibility for this case is the existence of similar components in the analyzed system. Suppose, for example, that components $A$ and $B$ are similar control valves. The state for which $A$ is failed and $B$ is on is quite similar to the one for which $A$ is on and $B$ is failed. These two states are eligible for merging and, thus, form a superstate. In this case, the lumpability or mergeability approach will not be useful as will be clear for the case study to be discussed in this paper, due to the lack of system symmetries.

To address the issue of modeling large systems, Takaragi et al. [6] proposed a method for reducing the number of states in the state transition diagram in a Markovian reliability analysis, making use of ineffective edges, which can be cut with little influence on the system failure probability, that is, in conceptual terms, to retain the most important states only.

Another model was presented by Gandini [7] who proposed to make the edge cuts using the Generalized Perturbation Theory (GPT), using as the integral quantity the probability of the system being in each state.

The objective of this work is to investigate an alternative way, which has as a theoretical basis the methodology employed by Takaragi et al. [6], which uses as an integral parameter the system average unavailability plus the methodology reported by Gandini [7], who proposed to make 


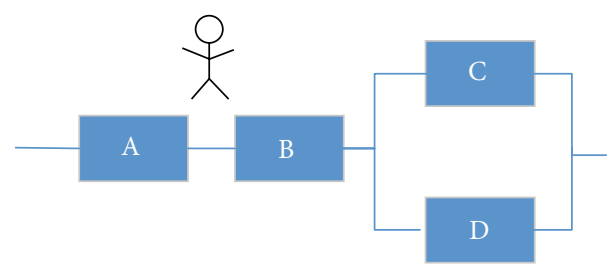

Figure 1: System logical diagram [6].

TABLE 1: Component parameters for the analysis [6].

\begin{tabular}{lcc}
\hline Component & Failure rate $\left(\mathrm{hr}^{-1}\right)$ & Repair rate $\left(\mathrm{hr}^{-1}\right)$ \\
\hline A & $\lambda_{1}=1.0 \times 10^{-5}$ & $\mu_{1}=1.0 \times 10^{-2}$ \\
B & $\lambda_{2}=8.0 \times 10^{-5}$ & $\mu_{2}=1.0 \times 10^{-2}$ \\
C & $\lambda_{3}=1.0 \times 10^{-4}$ & $\mu_{3}=1.0 \times 10^{-2}$ \\
D & $\lambda_{4}=5.0 \times 10^{-4}$ & $\mu_{4}=2.0 \times 10^{-3}$ \\
\hline
\end{tabular}

the edge cut study considering GPT, using the importance concept and obtaining the derivatives that will aid in cutting the less unimportant edges and simplifying the system state transition diagram.

In an earlier paper, we have discussed the application of the Generalized Perturbation Theory to the sensitivity analysis of the accident rate of plants equipped with protective channel systems [8].

This paper is organized as follows. Section 2 discusses the case study and its Markovian model, considering all relevant features, including adopted repair policies. Section 3 is dedicated to the discussion of how GPT can be applied to simplify the state transition diagram. The results obtained are discussed in Section 4 and the simplified transition diagram is presented and the results compared with those of the original transition diagram. Finally, Section 5 displays the conclusions reached.

\section{The Markovian Model and the Model Case}

The starting point for using the GPT formalism is to set the state transition diagram for the Markovian analysis. Figure 1 [6] displays the system block diagram whose relevant features are as follows:

(a) system has four components/pumps;

(b) there is a series system with two components/pumps and a parallel system also with two components/ pumps and both systems communicate;

(c) there is one repairman available;

(d) component failure rates are much smaller than their repair rates;

(e) components $A$ and $B$ have a higher repair priority than components $C$ and $D$;

(f) the repair strategy adopted for components $A$ and $B$ and $C$ and $D$ is first in, first out.

Table 1 [6] displays the parameters for the analysis.

Figure 2 displays the system state transition diagram [6]. State number 1 means that all system components are on and there is no system degradation. The next four states (2, 3, 4, and 5) display one failed component each. Each failed component is identified by the grey color. The third column displays the next degradation level for which two components are down. In this case, nine failed states are generated, those from 6 to 14. The fourth column displays the next system degradation level for which three out of four components are failed. All of them are subject to repair but there can be no superposition between subject to repair and repair priority required between components $C$ and $D$. Thus, seven possible states are generated (from 15 to 21). Finally, for the last degradation level all four components are failed. The only difference is the position variation between subject to repair and required repair priority, which generates four states (from 22 to 25 ).

According to the state transition diagram of Figure 2 one obtains the following system of coupled ordinary differential equations:

$$
\begin{aligned}
& \frac{d p_{1}(t)}{d t}=-\left(\lambda_{1}+\lambda_{2}+\lambda_{3}+\lambda_{4}\right) p_{1}(t)+\mu_{4} p_{2}(t) \\
& +\mu_{3} p_{3}(t)+\mu_{2} p_{4}(t)+\mu_{1} p_{5}(t) \\
& \frac{d p_{2}(t)}{d t}=\lambda_{4} p_{1}(t)-\left(\mu_{4}+\lambda_{1}+\lambda_{2}+\lambda_{3}\right) p_{2}(t) \\
& +\mu_{3} p_{7}(t)+\mu_{2} p_{8}(t)+\mu_{1} p_{10}(t) \\
& \frac{d p_{3}(t)}{d t}=\lambda_{3} p_{1}(t)-\left(\mu_{3}+\lambda_{1}+\lambda_{2}+\lambda_{4}\right) p_{3}(t) \\
& +\mu_{4} p_{6}(t)+\mu_{2} p_{9}(t)+\mu_{1} p_{11}(t) \\
& \frac{d p_{4}(t)}{d t}=\lambda_{2} p_{1}(t)-\left(\mu_{2}+\lambda_{1}+\lambda_{3}+2 \lambda_{4}\right) p_{4}(t) \\
& +\mu_{4} p_{12}(t)+\mu_{1} p_{13}(t) \\
& \frac{d p_{5}(t)}{d t}=\lambda_{1} p_{1}(t)-\left(\mu_{1}+2 \lambda_{2}+\lambda_{3}+\lambda_{4}\right) p_{5}(t) \\
& +\mu_{2} p_{14}(t) \\
& \frac{d p_{6}(t)}{d t}=\lambda_{3} p_{2}(t)-\left(\mu_{4}+\lambda_{1}+\lambda_{2}\right) p_{6}(t) \\
& +\mu_{1} p_{19}(t)+\mu_{2} p_{21}(t) \\
& \frac{d p_{7}(t)}{d t}=\lambda_{4} p_{3}(t)-\left(\mu_{3}+\lambda_{1}+\lambda_{2}\right) p_{7}(t) \\
& +\mu_{1} p_{18}(t)+\mu_{2} p_{20}(t) \\
& \frac{d p_{8}(t)}{d t}=\lambda_{2} p_{2}(t)+\lambda_{4} p_{4}(t)-\left(\mu_{2}+\lambda_{3}\right) p_{8}(t) \\
& +\mu_{1} p_{17}(t) \\
& \frac{d p_{9}(t)}{d t}=\lambda_{2} p_{3}(t)+\lambda_{3} p_{4}(t)-\left(\mu_{2}+\lambda_{4}\right) p_{9}(t), \\
& \frac{d p_{10}(t)}{d t}=\lambda_{1} p_{2}(t)+\lambda_{4} p_{5}(t)-\left(\mu_{1}+\lambda_{2}+\lambda_{3}\right) p_{10}(t),
\end{aligned}
$$




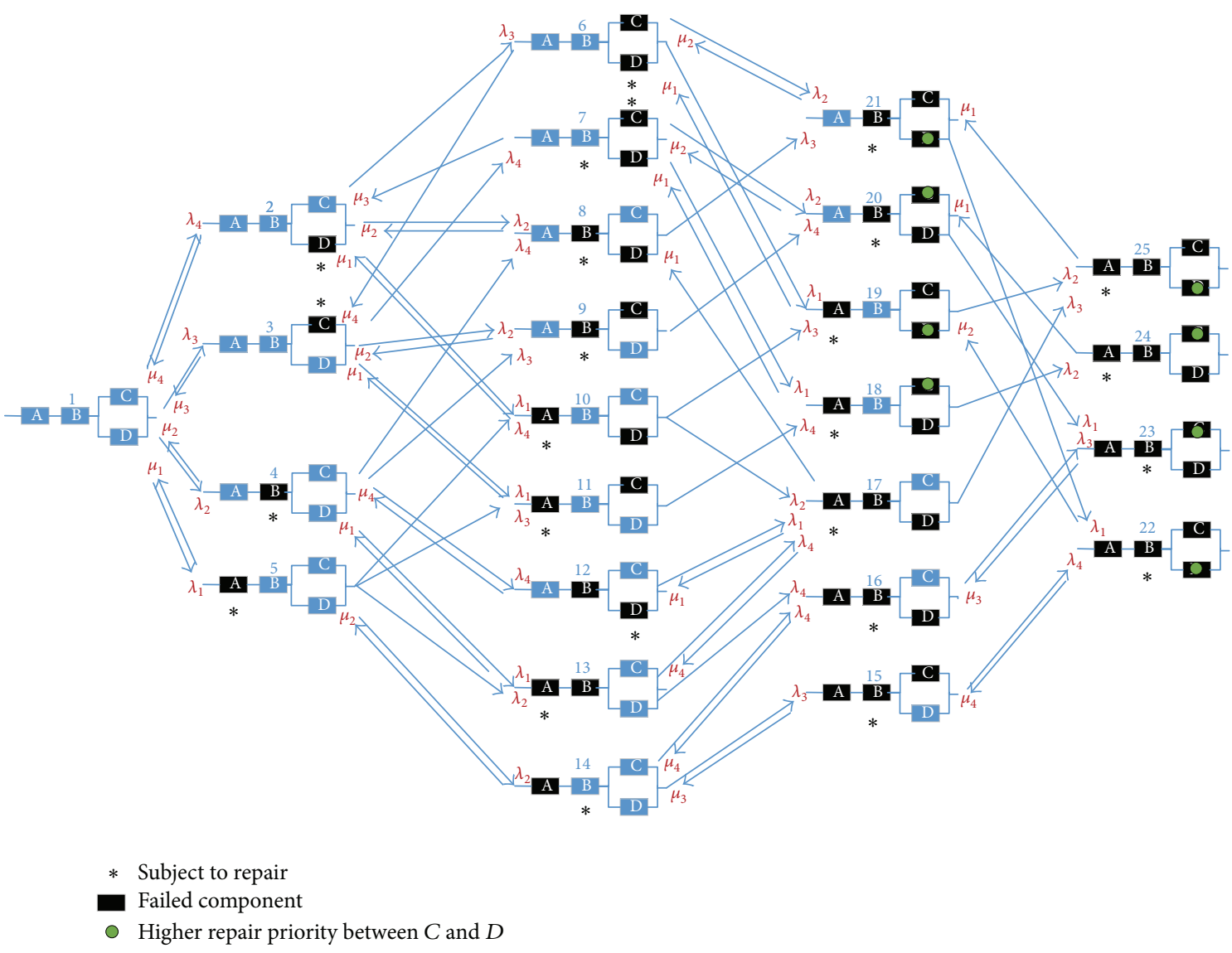

FIGURE 2: Markov state transition diagram: four-component system with a repairman.

$\frac{d p_{11}(t)}{d t}=\lambda_{1} p_{3}(t)+\lambda_{3} p_{5}(t)-\left(\mu_{1}+\lambda_{4}\right) p_{11}(t)$

$\frac{d p_{12}(t)}{d t}=\lambda_{4} p_{4}(t)-\left(\mu_{4}+\lambda_{1}\right) p_{12}(t)+\mu_{1} p_{17}(t)$,

$\frac{d p_{13}(t)}{d t}=\lambda_{1} p_{4}(t)+\lambda_{2} p_{5}(t)-\left(\mu_{1}+2 \lambda_{4}\right) p_{13}(t)$

$$
+\mu_{4} p_{17}(t)
$$

$\frac{d p_{14}(t)}{d t}=\lambda_{2} p_{5}(t)-\left(\mu_{2}+\lambda_{3}+\lambda_{4}\right) p_{14}(t)$

$+\mu_{3} p_{15}(t)+\mu_{4} p_{16}(t)$,

$\frac{d p_{15}(t)}{d t}=\lambda_{3} p_{14}(t)-\left(\mu_{3}+\lambda_{4}\right) p_{14}(t)+\mu_{4} p_{22}(t)$,

$\frac{d p_{16}(t)}{d t}=\lambda_{4} p_{13}(t)+\lambda_{4} p_{14}(t)-\left(\mu_{4}+\lambda_{3}\right) p_{16}(t)$

$$
+\mu_{3} p_{23}(t)
$$

$\frac{d p_{17}(t)}{d t}=\lambda_{2} p_{10}(t)+\lambda_{1} p_{12}(t)+\lambda_{4} p_{13}(t)$

$$
-\left(2 \mu_{1}+\mu_{4}+\lambda_{3}\right) p_{17}(t)
$$

$\frac{d p_{18}(t)}{d t}=\lambda_{1} p_{7}(t)+\lambda_{4} p_{11}(t)-\left(\mu_{1}+\lambda_{2}\right) p_{18}(t)$,

$\frac{d p_{19}(t)}{d t}=\lambda_{1} p_{6}(t)+\lambda_{3} p_{10}(t)-\left(\mu_{1}+\lambda_{2}\right) p_{19}(t)$

$+\mu_{2} p_{22}(t)$,

$\frac{d p_{20}(t)}{d t}=\lambda_{2} p_{7}(t)+\lambda_{4} p_{9}(t)-\left(\mu_{2}+\lambda_{1}\right) p_{20}(t)+\mu_{1} p_{24}(t)$,

$\frac{d p_{21}(t)}{d t}=\lambda_{2} p_{6}(t)+\lambda_{3} p_{8}(t)-\left(\mu_{2}+\lambda_{1}\right) p_{21}(t)+\mu_{1} p_{25}(t)$,

$\frac{d p_{22}(t)}{d t}=\lambda_{4} p_{15}(t)+\lambda_{1} p_{21}(t)-\left(\mu_{2}+\mu_{4}\right) p_{22}(t)$,

$\frac{d p_{23}(t)}{d t}=\lambda_{3} p_{16}(t)+\lambda_{1} p_{20}(t)-\left(\mu_{3}\right) p_{23}(t)$,

$\frac{d p_{24}(t)}{d t}=\lambda_{2} p_{18}(t)-\left(\mu_{1}\right) p_{24}(t)$,

$\frac{d p_{25}(t)}{d t}=\lambda_{3} p_{17}(t)+\lambda_{2} p_{19}(t)-\left(\mu_{1}\right) p_{25}(t)$. 
The system represented by (1) can be recast into matrix form as

$$
\frac{d \underset{\sim}{p}(t)}{d t}=M \underset{\sim}{p}(t),
$$

where $\underset{\sim}{p}(t)$ is the vector given by

$$
\underset{\sim}{p}(t)=\left[\begin{array}{lll}
p_{1}(t) & \cdots & p_{25}(t)
\end{array}\right]^{T}
$$

for which $p_{1}(t), \ldots, p_{25}(t)$ are the probabilities of being in each of the 25 defined system states.

The initial condition used for solving $(1)$ is $p_{1}(0)=1$ and $p_{i}(0)=0$ for $i=2, \ldots, 25$, which can be rewritten as

$$
\underline{\sim}(0)=\left[\begin{array}{llll}
1 & 0 & \cdots & 0
\end{array}\right]^{T} \text {. }
$$

This initial condition means that all system components are working at the beginning of system mission. Also, $M$ is the system transition rate matrix whose nonzero entries are defined as follows:

$M=\left[\begin{array}{cccccccccccc}a^{\prime} & \mu_{4} & \mu_{3} & \mu_{2} & \mu_{1} & 0 & 0 & 0 & 0 & \ldots & \ldots & 0 \\ \lambda_{4} & b^{\prime} & 0 & & & & & & & & & \\ \lambda_{3} & 0 & c^{\prime} & & & & & & & & & \\ \lambda_{2} & 0 & & d^{\prime} & & & & & & & & \\ \lambda_{1} & 0 & & & \ddots & & & & & & & \\ 0 & \lambda_{3} & & & & \ddots & & & & & & \\ 0 & 0 & & & & & \ddots & & & & & \\ 0 & \lambda_{2} & & & & & & \ddots & & & \\ \vdots & 0 & & & & & & & \ddots & & \\ \vdots & \lambda_{1} & & & & & & & & \ddots & & \\ 0 & \vdots & & & & & & & & & \ddots & \\ 0 & 0 & 0 & 0 & 0 & 0 & 0 & \ldots & \ldots & 0 & 0 & w^{\prime}\end{array}\right]_{25 \times 25}$

$a^{\prime}=-\left(\lambda_{1}+\lambda_{2}+\lambda_{3}+\lambda_{4}\right)$,

$b^{\prime}=-\left(\mu_{4}+\lambda_{1}+\lambda_{2}+\lambda_{3}\right)$,

$c^{\prime}=-\left(\mu_{3}+\lambda_{1}+\lambda_{2}+\lambda_{4}\right)$

$w^{\prime}=-\left(\mu_{1}\right)$

It may be seen from the state transition diagram of Figure 2 that the system is working only when it is in states 1,2 , or 3 and that it is failed when it is in states from 4 to 25 . In this way, the system point unavailability may be calculated from

$$
\bar{A}(t)=\sum_{i=4}^{25} p_{i}(t) .
$$

For evaluating the system mean availability for a given mission period or time, one uses [9]:

$$
A_{\text {Mean }}=\frac{1}{T} \int_{0}^{T} A(t) d t
$$

where $A(t)$ is the system availability, that is, the probability that the system works satisfactorily at time $t$.

The system average unavailability will be given by

$$
\bar{A}_{\text {Mean }}=\frac{1}{T} \int_{0}^{T} \bar{A}(t) d t,
$$

where $\bar{A}(t)$ is given by (6), so that

$$
\bar{A}_{\text {Mean }} \equiv \frac{1}{T} \int_{0}^{T} \sum_{i=4}^{25} p_{i}(t) d t .
$$

We will use GPT formalism to simplify the state transition diagram and so reduce the number of coupled ordinary differential equations to be solved.

\section{Using GPT to Analyze the System Mean Unavailability}

The development used to obtain the derivatives that will assist in simplifying Markovian state transition models for reliability analysis is presented below. This will be done by using the GPT formalism developed by Gandini [10], with the response of interest (integral quantity) being the average system unavailability. Then, the average system unavailability defined by (9) can be written as

$$
Q=\int_{0}^{T} \underset{\sim}{h}(t)^{+^{T}} \underset{\sim}{p}(t) d t
$$

where $p(t)$, the probability vector, satisfies $(2)$ and $\underset{\sim}{\operatorname{h}}(t)^{+^{T}}$ is a function defined as

$$
\underset{\sim}{h}(t)^{+^{T}} \equiv \frac{1}{T}\left[\begin{array}{llllll}
0 & 0 & 0 & 1 & \cdots & 1
\end{array}\right] .
$$

In this sense, one can write, from (10),

$$
\frac{\partial Q}{\partial a_{i j}}=\int_{0}^{T}\left\{\frac{\partial{\underset{\sim}{h}}^{+}}{\partial a_{i j}} \underset{\sim}{p}(t)+{\underset{\sim}{h^{+}}}^{T} \frac{\partial \underset{\sim}{p}(t)}{\partial a_{i j}}\right\} d t,
$$

where $a_{i j}$ 's are the entries of matrix $M$ of (5).

As ${\underset{h}{h}}^{+}(t)$ does not depend on the transition matrix entries then $\partial \underset{\sim}{h^{+}} / \partial a_{i j}=\underset{\sim}{0}$, and (12) may be written as

$$
\frac{\partial Q}{\partial a_{i j}}=\int_{0}^{T} \underline{h}^{+^{T}} \frac{\partial \underline{\sim}(t)}{\partial a_{i j}} d t .
$$

Functions $\underset{\sim}{p}(t) / \partial a_{i j}$ may be obtained from (2) as follows:

$$
\frac{d}{d t}\left(\frac{\partial \underset{\sim}{p}(t)}{\partial a_{i j}}\right)=M\left(\frac{\partial \underset{\sim}{p}(t)}{\partial a_{i j}}\right)+\frac{\partial M}{\partial a_{i j}} \underset{\sim}{p}(t) .
$$

But, according to the GPT formalism, more specifically, according to the principle of importance conservation and also to the source reciprocity relationship [11], the integral quantity defined in (13) may be obtained from

$$
\frac{\partial Q}{\partial a_{i j}}=\int_{0}^{T}{\underset{\sim}{*^{*}}}^{*^{T}}(t) \frac{\partial M}{\partial a_{i j}} \underset{\sim}{p}(t) d t,
$$


where $p^{*}(t)$ is the importance function associated with the integral quantity $Q$ defined by (10), which is governed by the following equation:

$$
-\frac{d{\underset{\sim}{p}}^{*}(t)}{d t}=M^{T}{\underset{\sim}{p}}^{*}+{\underset{\sim}{h}}^{+}(t) .
$$
has

As the quantity in entry $a_{i j}$ is also in entry $a_{j j}$, then one

$$
\underset{\partial a_{i j}}{\sim} \underset{\sim}{\sim}(t)=\left[\begin{array}{c}
0 \\
\vdots \\
0 \\
-p_{j}(t) \\
0 \\
\vdots \\
0 \\
p_{j}(t) \\
0 \\
\vdots \\
0
\end{array}\right] \longleftarrow \text { row } j \quad \text { row } i>j,
$$

or

$$
\underset{\partial a_{i j}}{\sim} \underset{\sim}{p}(t)=\left[\begin{array}{c}
0 \\
\vdots \\
0 \\
p_{j}(t) \\
0 \\
\vdots \\
0 \\
-p_{j}(t) \\
0 \\
\vdots \\
0
\end{array}\right] \longleftarrow \text { row } i \quad \text { row } i<j .
$$

By putting (17) or (18) into (15), it follows that

$$
\frac{\partial Q}{\partial a_{i j}}=\int_{0}^{T} p_{j}(t)\left\{p_{i}^{*}(t)-p_{j}^{*}(t)\right\} d t .
$$

Thus, the derivative of the integral quantity of interest is calculated by means of GPT for nonzero elements $a_{i j}$ of the state transition matrix from which we can proceed to the reduction of the state transition diagram.

The cutoff criterion will be the same used in [7], noting that the integral quantity used is the average system unavailability and the direction of the inequality is the reverse of the one proposed in [7] due to the change in the integral quantity. Explicitly, one has for the system unavailability $Q$

$$
\frac{\partial Q}{\partial a_{i j}}<\frac{\partial Q}{\partial a_{i j}^{\prime}},
$$

where $a_{i j}$ and $a_{i j}^{\prime}$ are the repair and failure rates, respectively. According to [7], if one considers as the integral quantity the probability of being in each state $\left[Q=P_{i}(t)\right]$ the cutoff criterion is given by

$$
\frac{\partial Q}{\partial a_{i j}}>\frac{\partial Q}{\partial a_{i j}^{\prime}}
$$

We have adopted (20) as our cutoff criterion, instead of (21), because our integral quantity is the system unavailability and not the probability of being in a given success state.

\section{Results}

The integral quantity $Q$ (average system unavailability) was calculated for the whole state transition diagram (25 system states) for a period of 12,500 mission hours, having a value of $1.501 \times 10^{-2}$, the same as the one found in [6]. Importance calculations for the original transition diagram were also made. Finally, the derivatives that are to be used for establishing the cutoff criterion were also evaluated (Table 2). A total of 66 derivatives were calculated although not all of them were effectively used.

Note that the only transition cut that differs from the reference work is the cut made in state 10 , which is not performed in [6]. In this sense, we have obtained a simpler transition diagram having 9 states instead of the 10 originally obtained in [6]. Figure 3 shows the cut in the initial state transition diagram.

Once the reduced state transition diagram is obtained, which can be seen in Figure 3, the new state transition matrix is made up by the following states: $1,2,3,4,5,6,7,8$, and 12 and the new integral quantity of interest (average system unavailability) has the value $Q=1.469 \times 10^{-2}$ for the mission period of 12,500 hours. The set of differential equations for the reduced diagram is

$$
\begin{aligned}
\frac{d p_{1}(t)}{d t}= & -\left(\lambda_{1}+\lambda_{2}+\lambda_{3}+\lambda_{4}\right) p_{1}(t)+\mu_{4} p_{2}(t) \\
& +\mu_{3} p_{3}(t)+\mu_{2} p_{4}(t)+\mu_{1} p_{5}(t), \\
\frac{d p_{2}(t)}{d t}= & \lambda_{4} p_{1}(t)-\left(\mu_{4}+\lambda_{2}+\lambda_{3}\right) p_{2}(t) \\
& +\mu_{3} p_{7}(t)+\mu_{2} p_{8}(t), \\
\frac{d p_{3}(t)}{d t}= & \lambda_{3} p_{1}(t)-\left(\mu_{3}+\lambda_{4}\right) p_{3}(t)+\mu_{4} p_{6}(t), \\
\frac{d p_{4}(t)}{d t}= & \lambda_{2} p_{1}(t)-\left(\mu_{2}+2 \lambda_{4}\right) p_{4}(t)+\mu_{4} p_{12}(t), \\
\frac{d p_{5}(t)}{d t}= & \lambda_{1} p_{1}(t)-\left(\mu_{1}\right) p_{5}(t), \\
\frac{d p_{6}(t)}{d t}= & \lambda_{3} p_{2}(t)-\left(\mu_{4}\right) p_{6}(t), \\
\frac{d p_{7}(t)}{d t}= & \lambda_{4} p_{3}(t)-\left(\mu_{3}+\lambda_{1}+\lambda_{2}\right) p_{7}(t) \\
& +\mu_{1} p_{18}(t)+\mu_{2} p_{20}(t),
\end{aligned}
$$


TABLE 2: Derivatives for the edge cutoff criterion.

\begin{tabular}{|c|c|c|c|c|c|c|c|c|}
\hline Case & $\begin{array}{l}\text { Failure } \\
(j \rightarrow i)\end{array}$ & $\begin{array}{c}\text { Failure rate } \\
\lambda\left(\mathrm{hr}^{-1}\right)\end{array}$ & $\frac{\partial Q}{\partial a_{i j}^{\prime}}$ & $\begin{array}{c}\text { Repair } \\
(j \rightarrow i)\end{array}$ & $\begin{array}{c}\text { Repair rate } \\
\mu\left(\mathrm{hr}^{-1}\right)\end{array}$ & $\frac{\partial Q}{\partial a_{i j}}$ & $\begin{array}{c}\text { Condition } \\
\text { satisfied }\end{array}$ & Cut \\
\hline 1 & $2 \rightarrow 6$ & $1.0 E-4$ & $8.11 E-4$ & $\begin{array}{l}19 \rightarrow 6 \\
21 \rightarrow 6\end{array}$ & $\begin{array}{l}1.0 E-2 \\
2.0 E-2\end{array}$ & $\begin{array}{l}-4.03 E-8 \\
-7.29 E-8\end{array}$ & Yes & $\begin{array}{l}19 \rightarrow 6 \\
21 \rightarrow 6\end{array}$ \\
\hline 2 & $3 \rightarrow 7$ & $5.0 E-4$ & $1.66 E-4$ & $\begin{aligned} 20 & \rightarrow 7 \\
18 & \rightarrow 7\end{aligned}$ & $2.0 E-2$ & $\begin{array}{l}-5.75 E-9 \\
-4.56 E-9\end{array}$ & Yes & $\begin{array}{l}20 \rightarrow 7 \\
18 \rightarrow 7\end{array}$ \\
\hline 3 & $2 \rightarrow 8$ & $8.0 E-5$ & $3.49 E-4$ & $17 \rightarrow 8$ & $2.0 E-2$ & $5.57 E-9$ & Yes & $17 \rightarrow 8$ \\
\hline 4 & $1 \rightarrow 3$ & $1.0 E-4$ & $3.81 E-3$ & $9 \rightarrow 3$ & $2.0 E-2$ & $-1.20 E-7$ & Yes & $9 \rightarrow 3$ \\
\hline 5 & $1 \rightarrow 2$ & $5.0 E-4$ & $1.52 E-2$ & $10 \rightarrow 2$ & $1.0 E-2$ & $-8.03 E-7$ & Yes & $10 \rightarrow 2$ \\
\hline 6 & $1 \rightarrow 3$ & $1.0 E-4$ & $3.81 E-3$ & $11 \rightarrow 3$ & $1.0 E-2$ & $-7.35 E-8$ & Yes & $11 \rightarrow 3$ \\
\hline 7 & $4 \rightarrow 12$ & $5.0 E-4$ & $5.72 E-3$ & $17 \rightarrow 12$ & $1.0 E-2$ & $-6.25 E-9$ & Yes & $17 \rightarrow 12$ \\
\hline 8 & $1 \rightarrow 4$ & $8.0 E-5$ & $2.27 E-3$ & $13 \rightarrow 4$ & $1.0 E-2$ & $4.41 E-7$ & Yes & $13 \rightarrow 4$ \\
\hline 9 & $1 \rightarrow 5$ & $1.0 E-5$ & $3.52 E-3$ & $14 \rightarrow 5$ & $2.0 E-2$ & $-7.60 E-9$ & Yes & $14 \rightarrow 5$ \\
\hline
\end{tabular}

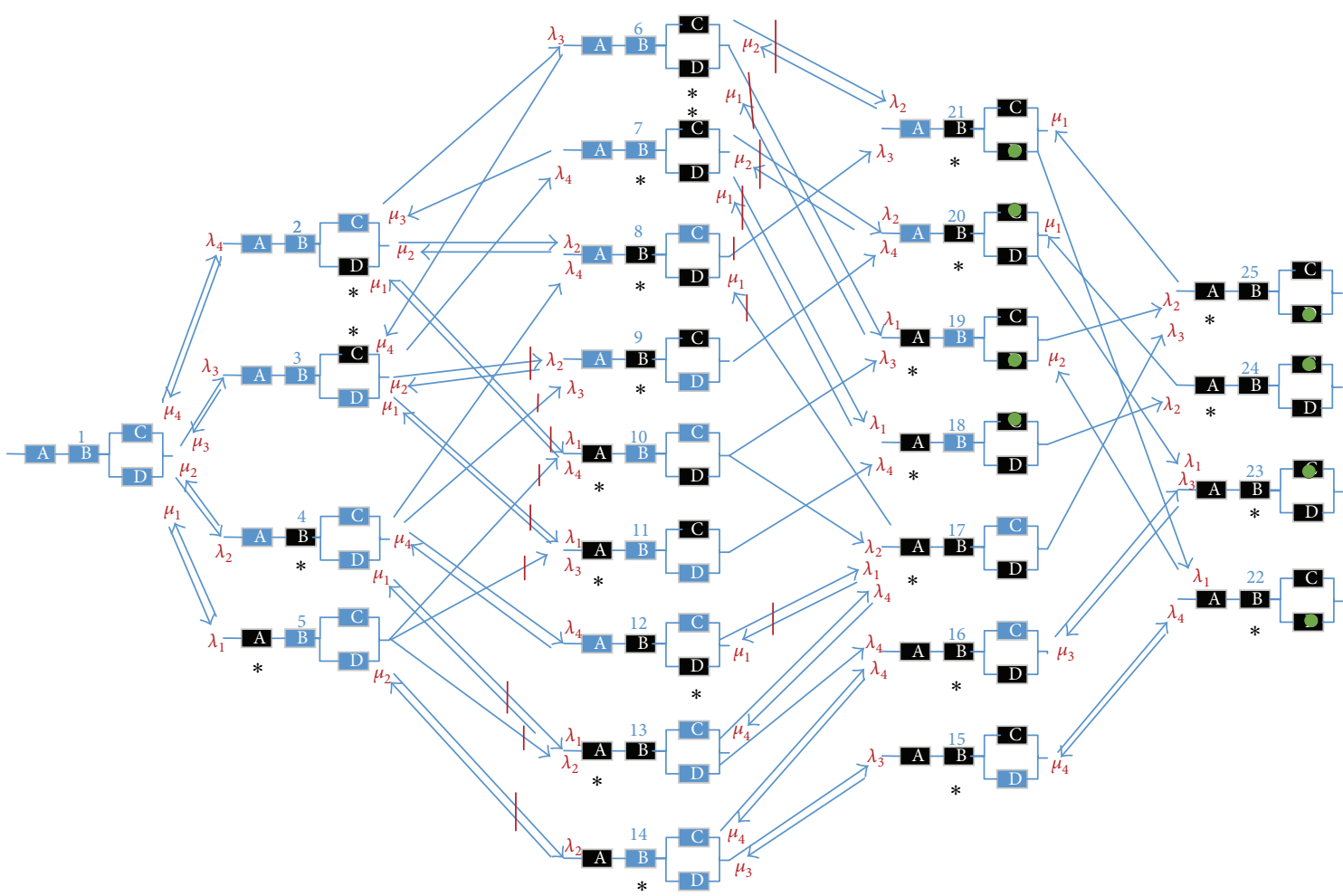

* Subject to repair

Failed component

- Higher repair priority between $C$ and $D$

FIGURE 3: State transition diagram with cut performed (retained states to the left of the red line).

$$
\begin{aligned}
& \frac{d p_{8}(t)}{d t}=\lambda_{2} p_{2}(t)+\lambda_{4} p_{4}(t)-\left(\mu_{2}\right) p_{8}(t) \\
& \frac{d p_{12}(t)}{d t}=\lambda_{4} p_{4}(t)-\left(\mu_{4}\right) p_{12}(t) .
\end{aligned}
$$

If, instead of using the original diagram with its average system unavailability $\left(Q=1.501 \times 10^{-2}\right)$, we use the average system unavailability for the simplified diagram, we would err by $2.18 \%$. Figure 4 shows the simplified state transition diagram.

In order to further investigate the relative error, we calculated the average system reliability considering the original state transition diagram with the 25 states and the simplified diagram for different mission times. Figure 5 displays the results. It may be seen that even considering mission times from 1.5 months up to about 3.5 years the relative error variation is $2.18 \%$ at most. 


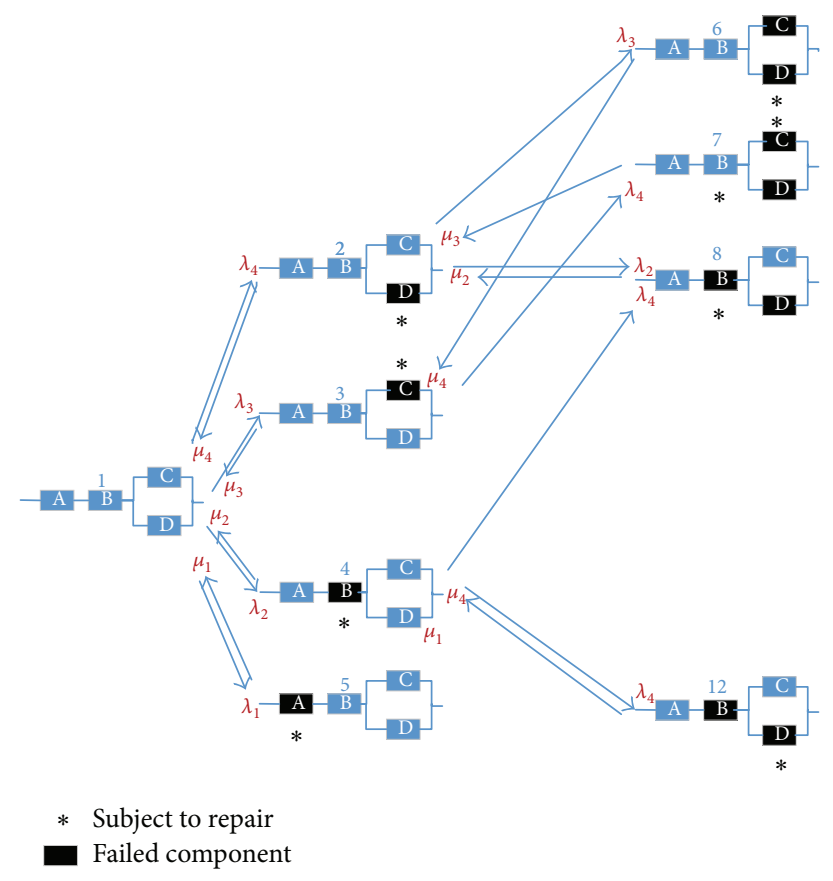

FIGURE 4: Simplified state transition diagram obtained by means of GPT.

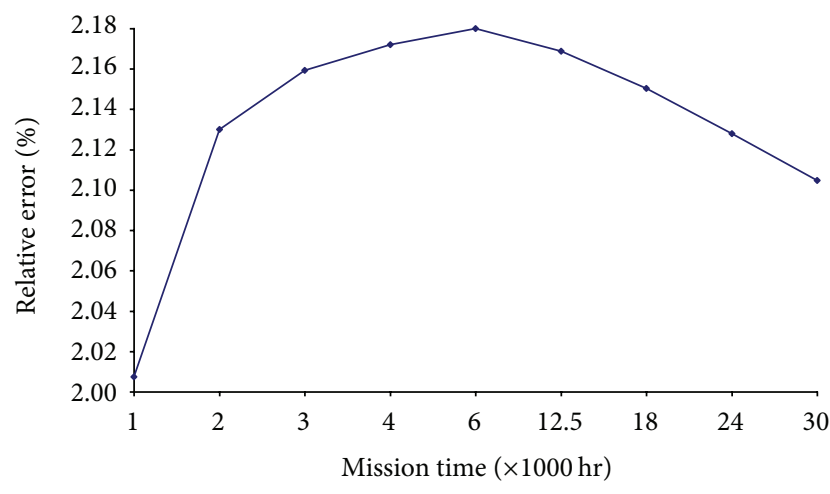

FIGURE 5: Variation of relative error with mission time.

\section{Conclusions}

A methodology to simplify state transition diagrams in Markovian reliability analysis that may have a lot of states was presented. The implementation of this methodology is relatively simple compared to that presented in [6].

In this study we analyzed the integral quantity of interest (average system unavailability) with constant failure and repair rates and considering the state transition diagram of the original 25 states. It was not considered in this study that at least one of the elements could have a time-dependent failure rate; that is, we have not considered aging. With the simplified transition diagram (9 states), the new value of the integral quantity of interest was very close to the original system.

The integral quantity of interest (system average unavailability) for a mission period of 12,500 hours considering the state transition diagram of the original 25 states was $Q=$ $1.501 \times 10^{-2}$. After simplifying the state transition diagram by means of GPT, the new average system unavailability was $\mathrm{Q}=1.469 \times 10^{-2}$.

It was shown that by using GPT formalism it was not possible to obtain the same edge criterion cut proposed in [6], which also contained state 10 and whose integral quantity is given by $Q=1.487 \times 10^{-2}$. The reason for this difference in the simplified transition diagram is a result of the change in the integral quantity since there may be states that have little influence when their derivatives with respect to failure and repair are evaluated for cutting the transition diagram, but in general the simplified transition diagram will be very close.

However, the proposed edge cut using the GPT formalism can be considered a good approximation of the original transition diagram for the calculation of $Q$, with a relative error of $2.1 \%$. By varying the system mission time, the maximum relative error obtained with the edge cutting procedure was $2.18 \%$.

Future studies may investigate whether the behavior of the cutoff criterion used in this work will be valid for other integral quantities of interest and will decrease the relative error. It is recommended to make a comparative study considering aging in which at least one of the components is undergoing aging, which will result in greater complexity for the systems of differential equations and new issues need to be considered and perhaps new cutoff proposals might have to be considered.

\section{Conflict of Interests}

The authors declare that there is no conflict of interests regarding the publication of this paper. 


\section{References}

[1] J. Endrenyi, Reliability Modeling in Electric Power Systems, Wiley, Chichester, UK, 1980.

[2] C. Singh and R. Billington, "Frequency and duration concepts in system reliability evaluation," IEEE Transactions on Reliability, vol. R-24, no. 1, pp. 31-36, 1975.

[3] J. G. Kemeny and J. L. Snell, Finite Markov Chains, Van Nostrand, Princeton, NJ, USA, 1960.

[4] I. A. Papazoglou and E. P. Gyftopoulos, "Markovian reliability analysis under uncertainty with an application on the shutdown system of the Clinch River Breeder reactor," Tech. Rep. NUREG/CR-0405, U. S. Nuclear Regulatory Commission, Washington, DC, USA, 1978.

[5] I. A. Papazoglou, "Elements of Markovian reliability analysis," in Reliability Engineering, A. Amendola and A. S. Saiz de Bustamante, Eds., pp. 171-204, Kluwer Academic Press, Dodrecht, The Netherlands, 1988.

[6] K. Takaragi, R. Sasaki, and S. Shingai, "A method of rapid Markov reliability calculation," IEEE Transactions on Reliability, vol. R-34, no. 3, pp. 262-268, 1985.

[7] A. Gandini, "System reliability assessment via sensitivity analysis," in Safety Design Criteria for Industrial Plants, M. Cuomo and A. Naviglio, Eds., pp. 121-135, CRC Press, Boca Ratton, Fla, USA, 1989.

[8] P. F. Frutuoso e Melo, A. C. M. Alvim, and F. C. Silva, "Sensitivity analysis on the accident rate of a plant equipped with a single protective channel by generalized perturbation methods," Annals of Nuclear Energy, vol. 25, no. 15, pp. 1191-1207, 1998.

[9] E. E. Lewis, Introduction to Reliability Engineering, Wiley, New York, NY, USA, 1996.

[10] A. Gandini, "Importance and sensitivity analysis in assessing system reliability," IEEE Transactions on Reliability, vol. 39, no. 1, pp. 61-70, 1990.

[11] A. Gandini, "Generalized Perturbation Theory (GPT) methods. A Heuristic approach," in Advances in Nuclear Science and Technology, J. Lewins and M. Becker, Eds., vol. 19, pp. 205-380, Plenum Press, New York, NY, USA. 


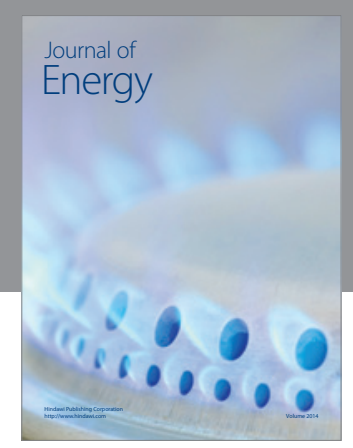

Journal of

Industrial Engineering
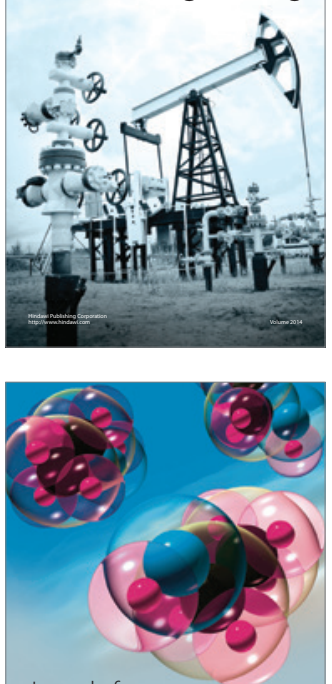

Fuels
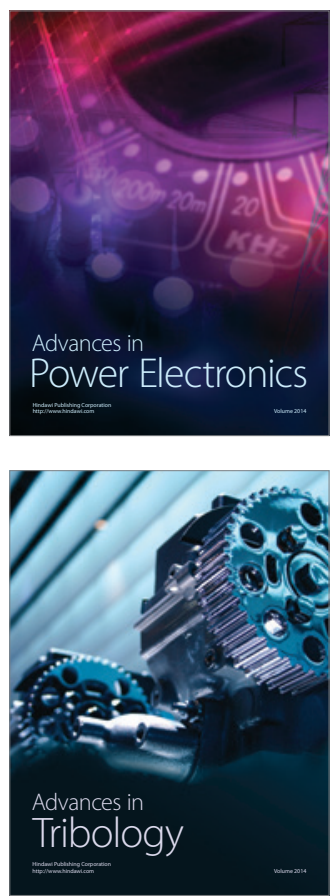

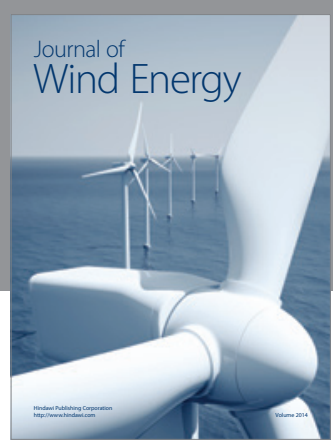

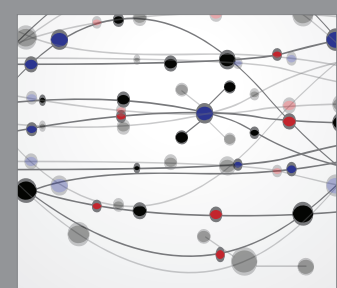

The Scientific World Journal

Submit your manuscripts at http://www.hindawi.com

Journal of

Structures
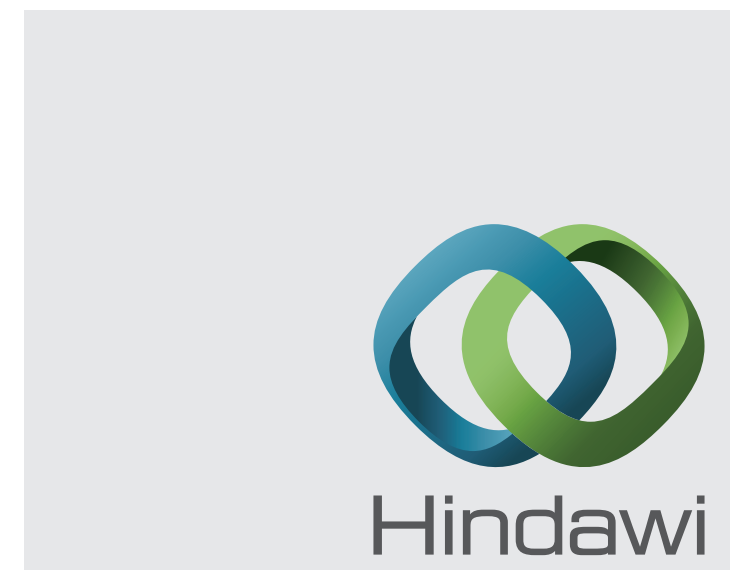

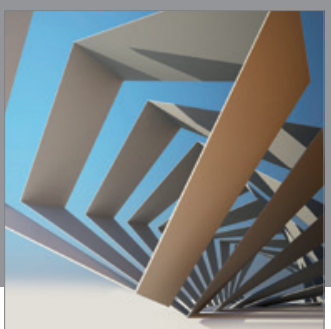

Rotating

Machinery
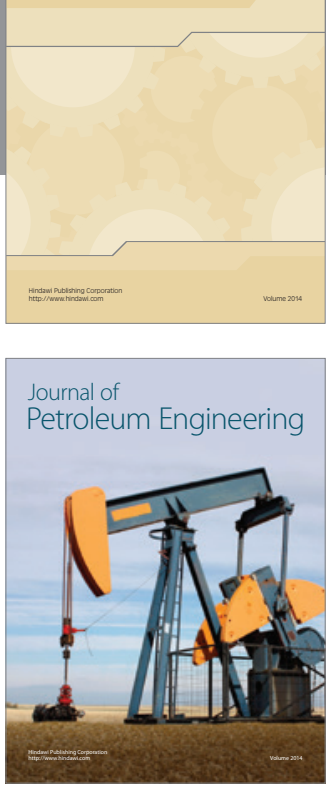

Journal of

Solar Energy
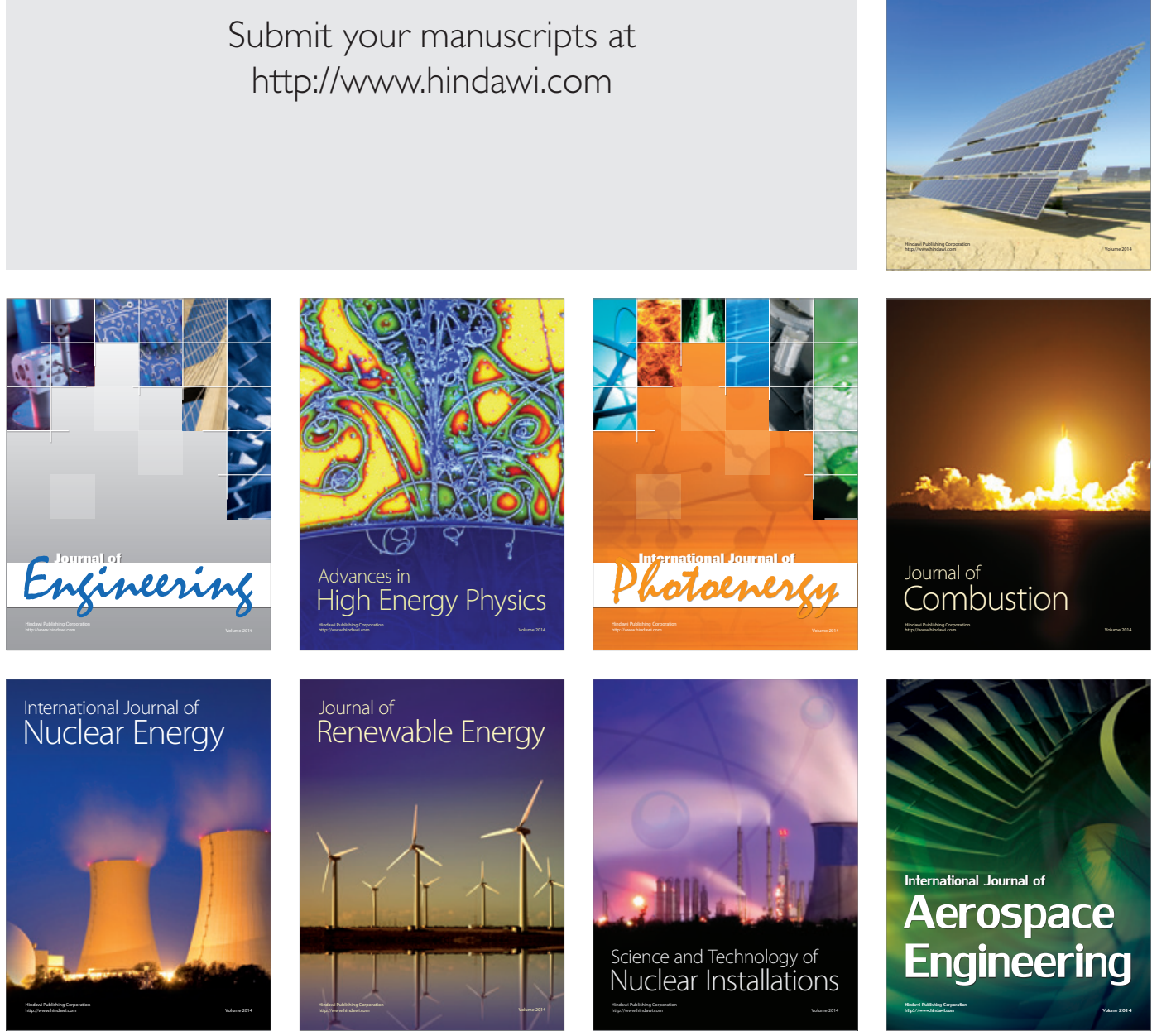